\title{
Routing of Terahertz Channels in Reconfigurable DWDM Digitally Switched Network
}

\author{
Aruna Rani ${ }^{1}$, Manjit Singh Bhamrah ${ }^{1}$, Sanjeev Dewra ${ }^{2}$ \\ ${ }^{1}$ Deptt. of ECE, Punjabi University, Patiala (INDIA) \\ ${ }^{2}$ Deptt. of ECE, Shaheed Bhagat Singh State Technical Campus, Ferozepur (INDIA) \\ Email: arunarani70@gmail.com, manjitsingh_manjit@rediffmail.com, sanjeevdewra09@gmail.com \\ Received 06.02.2021, Received in revised form 19.03.2021, Accepted 08.04.2021
}

\begin{abstract}
The reconfiguration in the dense wavelength division multiplexed transmission of 64 channels spaced on $100 \mathrm{GHz}$ channel spacing using digital switches is demonstrated. The system performance has been demonstrated by changing the power of input signal from $0 \mathrm{dBm}$ to $-20 \mathrm{dBm}$ and data rate from 20 Gbps to 40 Gbps. Transmission over $180 \mathrm{~km}$ distance at 40 Gbps data rate with $-10 \mathrm{dBm}$ input power is achieved. The behavior of the system is also analyzed by varying the attenuation from $0 \mathrm{~dB}$ to $30 \mathrm{~dB}$ of reconfigurable optical add drop multiplexer. The results have been reported for the DWDM system at the 193.1 $\mathrm{THz}$ (Ist channel) and $199.5 \mathrm{THz}$ (64 channel) in terms of OSNR, Q-factor and BER.
\end{abstract}

Keywords - ROADM, DWDM, data rate, digital switches, attenuation.

\section{INTRODUCTION}

The bandwidth of the network becomes critical when number of optical channels is being established. In an optical network, add or drop different channels is essential and optical add/drop multiplexer is the significant element to facilitate more flexibility and connectivity to the network where the quantity of wavelengths in the network and their provision arrangements are static and prearranged. This executes a major drawback on facility suppliers in managing of the optical networks [1]. The core challenges to construct the dynamically provisioned network for future are flexibility and slow services. On the other hand, ROADM is a real world methodology to remotely adjusting the channels to be added/dropped or delivered over a node. The power consumption and system cost becomes impracticable as the quantity of wavelengths rise or passing on other signal sources at every node [2]. ROADM allows several wavelengths to be dropped or added from the fiber without O-E-O conversion $[3,4]$. The key benefits of ROADM in the systems are the capability to assign the existing system bandwidth to specific users whereas without disturbing the stream of traffic and stable the power achieved by each ROADM [5]. The network constructed on ROADM is beneficial for transferring or progression the current optical networks.
ROADM has flexibility on the provisioning of channels irrespective in what manner the network changes [6]. ROADM enhances the flexibility of a network and becomes an easy and remotely achieved method providing full control [7]. C.A. Sayeed et al. [8] proposed a hybrid ROADM having $7 \mathrm{~dB}$ insertion loss. The signals were transmitted at 2.48 Gbps data rate. This ROADM have limited low loss designs that will allow considerably larger distances maximum $65 \mathrm{~km}$. Vahid Abedifar et al. [9] proposed a mesh topology with five node ROADM architecture. The BER of channels was varied by changing the added/dropped channels in intermediary nodes. It is necessary to achieve drop/add of wavelengths exactly and recompense its influence on the quality of other channels. This was attained through adjustable attenuators in particular positions of light path. Mark Filer and Sorin Tibuleac [10] proposed the WSS components in ROADM networks at 40 Gbps operating on the 50 GHz. The development of low cost WSS technology is allowing ROADMs to extend into long haul systems. Brigitte Jaumard and D.T. Kien [11] proposed a ROADM model for the best routing comparability to enhance the grade of service for a specific number of ports. This can differ expressively up to $30 \%$ dependent on the transferring connectivity. Wavelength switched optical networks had been planned for high efficiency to take advantage of all optical switching fabrics. The WSS indicate the important switching components with a technology supporting multi-degree ROADM designs with colorless, directionless and contentionless switching. Kyriakos Vlachos [12] analyzed the architecture of ROADM with QAM signals. The configuration was depending on the structure of modular interferometer, FFT/IFFT filtering and sampling of time domain. The scaling capability had been presented up to offset 32-QAM. Sanjeev Dewra [13] investigated the crosstalk effect of ROADM based on DCE with 40 channels at 10 Gbps data rate and $100 \mathrm{GHz}$ channel spacing. It was found that the signal can be communicated up to 220 $\mathrm{km}$ at $15 \mathrm{~dB}$ crosstalk with the output power of -40 $\mathrm{dBm}$. The results presented in $[8,13]$ is limited to bit rate and transmission distance. We have extended the 
work to 40 Gbps with enhancement in the optical distance using ROADM.

The manuscript is structured as follows: The details of the system set up and ROADM structure is analyzed in the section 2 . The system performance is calculated in section 3 . The conclusion of this work is presented in section.

\section{SIMULATIVE ANALYSIS}

The 64 channels DWDM system performance with ROADM is analyzed. Fig. 1 describes the block diagram of optical system.

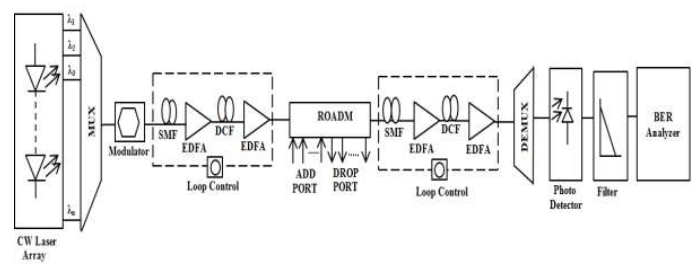

Fig.1 Functional block diagram of the DWDM System

The wavelength is selected between $193.1 \mathrm{THz}$ to $199.4 \mathrm{THz}$ of $100 \mathrm{GHz}$ in the planned DWDM system. The signal having wavelength $193.1 \mathrm{THz}$ to 196.2 $\mathrm{THz}$ is multiplexed and passed through the modulator. The modulated signal is passed through the combination of SMF having length of $50 \mathrm{~km}$ and DCF of $10 \mathrm{~km}$. The DCF is used at the end of SMF before applied to the ROADM to compensate the dispersion effect.

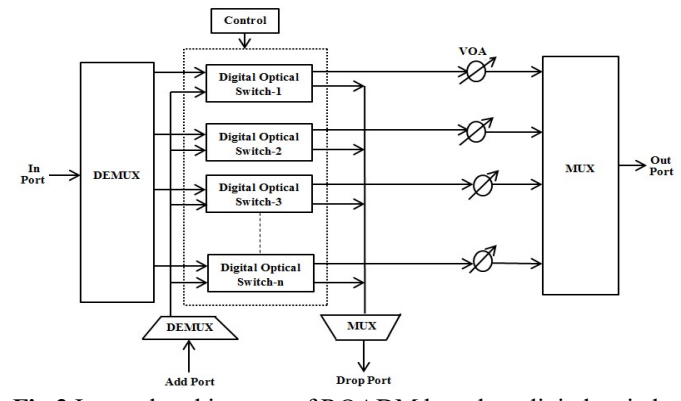

Fig.2 Internal architecture of ROADM based on digital switches

The design of ROADM is depicted in fig. 2 . ROADM is employed to drop/add and switch the wavelengths. ROADM is construct using digital switches, multiplexer and demultiplexer. The digital switches permits distinct or several channels to be dropped and/or added from a fiber without O-E-O conversion. The 32 wavelengths range from 196.3 $\mathrm{THz}$ to $199.4 \mathrm{THz}$ is added to add port of the ROADM. The demultiplexer is used to demultiplex these wavelengths and passed through the digital switches. The digital switch directs the signals in accordance with control signal at in port and to out ports. The optical signal at in port is passed to out port when the control signal is 0 and the wavelengths at add port is received at the drop port. If the control signal is 1 , then the wavelengths at input port are dropped and the added wavelengths are passed through the out port. It is essential to achieve drop/add of channels correctly and recompense the effect on the other channel quality. The ROADM adds and drops the group of wavelengths (band) dynamically. After the variable attenuators, demultiplexed channels are applied to BER analyzer for investigating the performance.

\section{RESULTS AND DISCUSSION}

The quality factor versus input power is shown in fig. 3. It is found that quality factor decreases when the bit rate rises from 20 to $40 \mathrm{Gbps}$. The quality factor varies from 96.58 to 25.82 for $20 \mathrm{Gbps}, 61.20$ to 18.39 for $30 \mathrm{Gbps}$ and 30.56 to 14.39 for $40 \mathrm{Gbps}$ respectively as the power is changed from 0 to -20 $\mathrm{dBm}$.

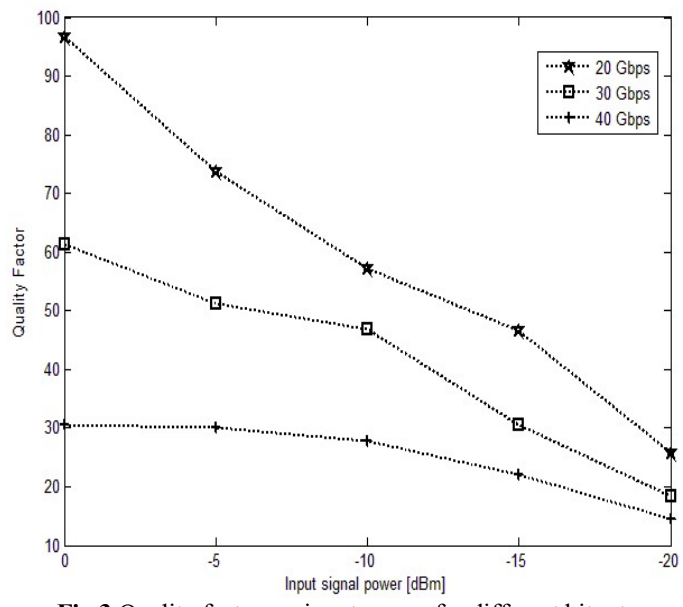

Fig.3 Quality factor vs. input power for different bit rates

The BER vs. power at different bit rate is shown in fig. 4. It is found that BER increases when the bit rate increases from $20 \mathrm{Gbps}$ to $40 \mathrm{Gbps}$ and input power decreases from 0 to $-20 \mathrm{dBm}$.

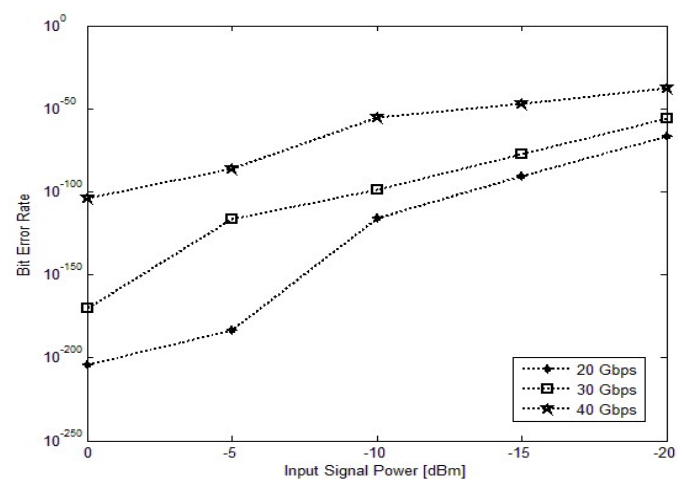

Fig.4 Bit error rate vs. input power for different bit rates 


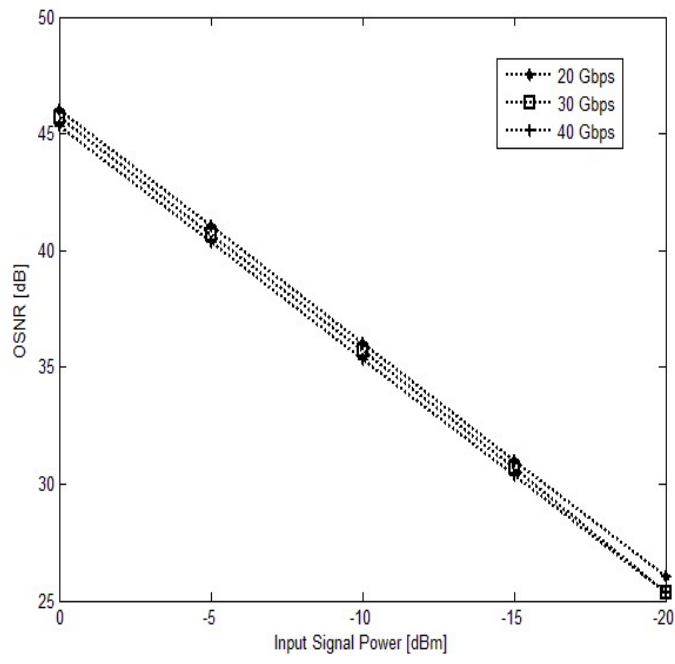

Fig.5 OSNR vs. input power for different bit rates

The OSNR vs. input power for 64 channels at different bit rates is depicted in graph 5 . It is found from the graph that as the power of input signal reduces, the value of OSNR also reduces. The OSNR varies from 46.03 to 26.03 for $20 \mathrm{Gbps}, 45.68$ to 25.35 for $30 \mathrm{Gbps}$ and 45.35 to 25.35 for $40 \mathrm{Gbps}$ respectively as the input power of a signal is varied from 0 to $-20 \mathrm{dBm}$.

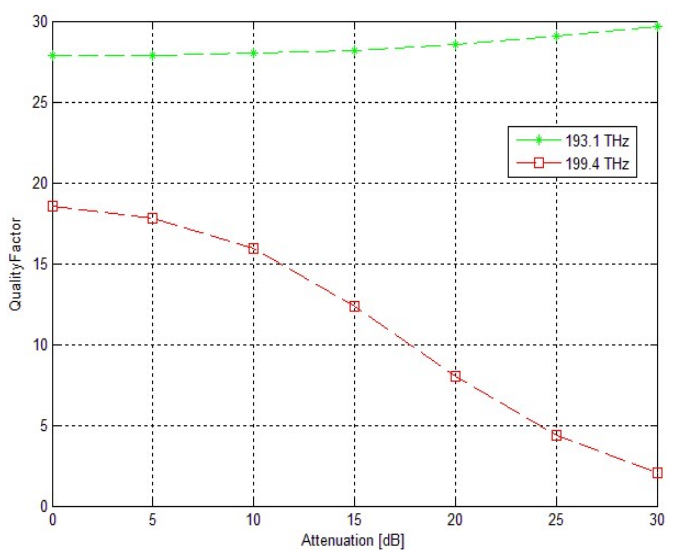

Fig.6 Quality factor vs. attenuation at $193.1 \mathrm{THz} \& 199.4 \mathrm{THz}$

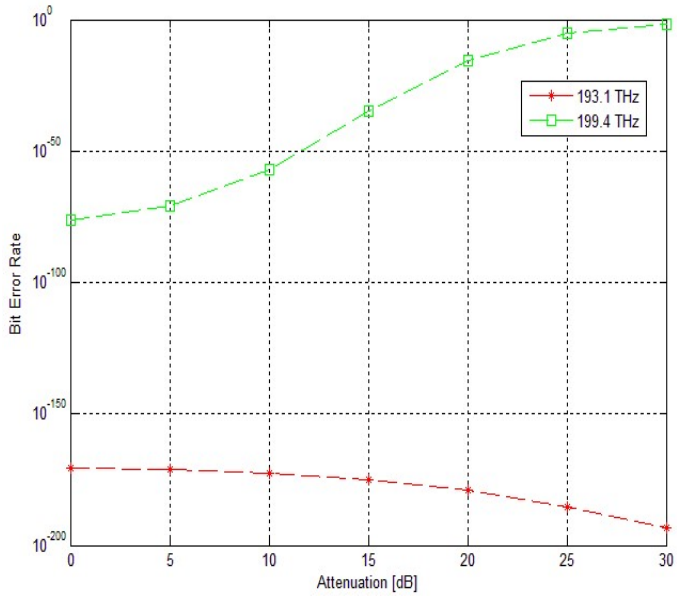

Fig.7 Bit error rate vs. attenuation at $193.1 \mathrm{THz}$ and $199.4 \mathrm{THz}$
The Q-factor and BER is shown in figure 6 and 7 by changing the attenuation. The value of the Q-factor and BER is calculated at the first channel (193.1 $\mathrm{THz})$ and last channel (199.5 $\mathrm{THz})$ by varying the attenuation factor of a ROADM from 0 to $30 \mathrm{~dB}$. As the attenuation increases, the quality factor reduces and BER rises. The quality factor is 27.84 to 29.6736 at $193.1 \mathrm{THz}$ frequency and 18.528 to 2.04897 at $199.5 \mathrm{THz}$. As the attenuation increases from $0 \mathrm{~dB}$ to $30 \mathrm{~dB}$, the bit error rate varies from $3.27 \mathrm{e}-171$ to $4.29 \mathrm{e}-194$ for channel-1 and $3.35 \mathrm{e}-77$ to 0.017362 for channel 64.

The optical spectrum at (a) transmitter (b) receiver side is shown in figure 8 . This graph depicts the power variations at different wavelengths. It shows the changes in output power for varying the input signal power and bit rate.
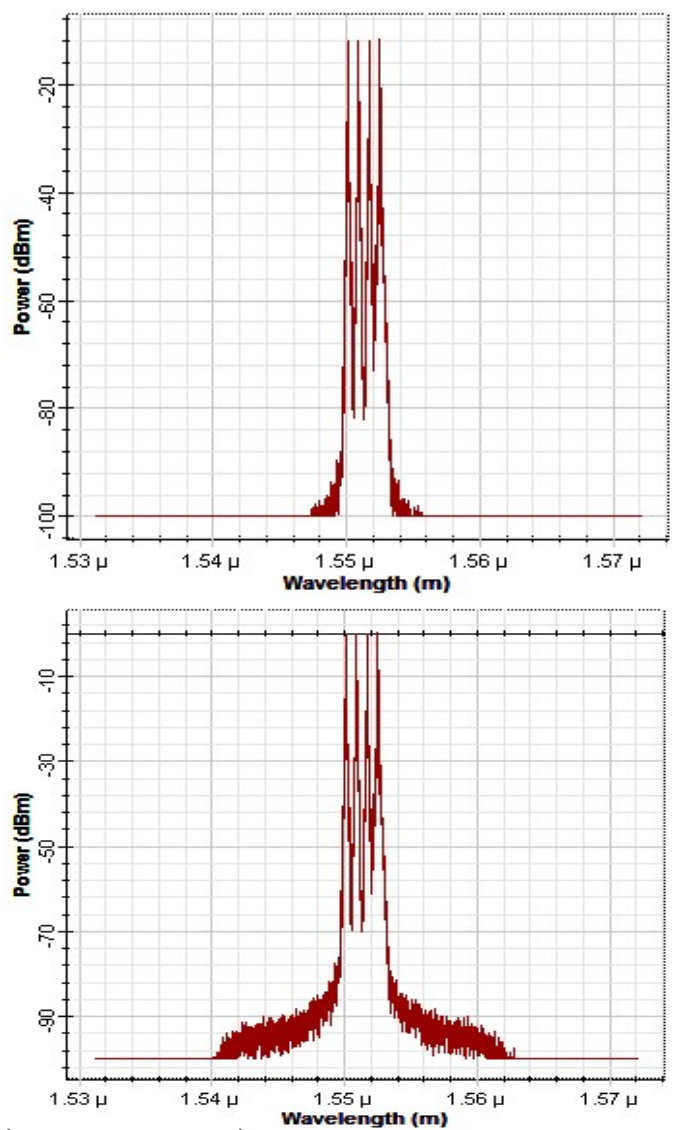

Fig. 8: Optical spectrum at transmitter (top) and receiver side (bottom)

\section{CONCLUSION}

The performance and feasilbility of DWDM system based on ROADM is presented in this paper. The signal of $-10 \mathrm{dBm}$ power can be communicated up to $180 \mathrm{~km}$ at $40 \mathrm{Gbps}$ data rate. The outcomes have been reported for the DWDM system having transmitting power from 0 to $-20 \mathrm{dBm}$. The quality factor and BER is calculated at the first channel 
(193.1 THz) and last channel (199.5 THz) by varying the attenuation factor of a ROADM. As the attenuation increases, quality factor of a signal reduces and BER rises. The design of the ROADM based on digital optical switches is analyzed to be beneficial as the key establishment in today's communications.

\section{ACKNOWLEDGMENT}

Authors wish to convey thanks to Ministry of Electronics and Information Technology, Government of India, for Visvesvaraya Ph.D scheme. This work is supported by visvesvaraya $\mathrm{PhD}$ scheme for Electronics \& IT, Meity, India. The unique awardee number is VISPHD-MEITY-1678.

\section{REFERENCES}

[1] G. Kenya Suzuki, Kazunori Seno and Yuichiro Ikuma, "Application of waveguide/free space optics hybrid to ROADM device," Journal of lightwave technology, ( 2017) ,Volume 35, no.4, Ppages 596-606.

[2] Jonathan Homa, Krishna Bala, "ROADM architectures and their enabling WSS technology," IEEE communications Magazine,( 2008),Volume 46, no. 7, Pages 150-154, 2008.

[3] Diogo G. Sequeira, Luís G. Cancela and João L. Rebola, "Impact of physical layer impairments on multi-degree CDC ROADM based optical networks", International conference on optical network design and modeling, (2018,) Page 94-99.

[4] Rani, A., Bhamrah, M.S. \& Dewra, S. Performance evaluation of the dense wavelength division multiplexing system using reconfigurable optical add/drop multiplexer based on digital switches. Opt Quant Electron 52, 480 (2020). https://doi.org/10.1007/s11082-020-02608-x.

[5] An Vu Tran, Wen De Zhong, Rodney S. Tucker, Kai Song, "Reconfigurable multichannel optical add drop multiplexers incorporating eight-port optical circulators and fibre bragg gratings," IEEE photonics technology letters, (2001), Volume 13, no. 10, Pages 1100-1102.

[6] V.A.J.M. Sleiffer, D. van den Borne, V. Veljanovski, M. Kuschnerov, M. Hirano, Y. Yamamoto, T. Sasaki, S.L. Jansen, and H. de Waardt, "Transmission of $448 \mathrm{~Gb} / \mathrm{s}$ dual carrier POLMUX 16 QAM over $1230 \mathrm{~km}$ with 5 flexi-grid ROADM passes," OFC/NFOEC technical digest, OSA, (2012).

[7] Jane M. Simmons, Adel A. M. Saleh, "Wavelength selective CDC ROADM designs using reduced sized optical cross connects", IEEE photonics technology letters, (2015), Volume 27, no.20,Pages $2174-2177$.

[8] C.A. Al Sayeed, A. Vukovic, O.W.W. Yang and Heng Hua, "Low loss reconfigurable OADM for metro core optical network," IET Optoelectronics (2007),Volume 1, no. 4, Pages 178-184.

[9] Vahid Abedifar, Sadegh Abbasi Shahkooh, Ali Emami, Ali Poureslami, S. A. Ayoughi, "Design and simulation of a ROADM based DWDM Network," 21st Iranian conference on Electrical Engineering (ICEE),(2013). pp.1-4.

[10] Sorin Tibuleac and Mark Filer, "Transmission impairments in DWDM networks with reconfigurable optical add drop multiplexers," Journal of lightwave technology, (2010) ,Volume 28, no. 4.

[11] Brigitte Jaumard, D.T. Kien, "Optimizing ROADM configuration in WDM networks," 16th International telecommunications network strategy and planning symposium network, (2014),Pages 1-7.

[12] Kyriakos Vlachos, Filipe Ferreira, Stelios Sygletos, "Performance evaluation of a reconfigurable optical add drop multiplexer design for high order regular and offset QAM signals," 20th International conference on transparent optical networks, (2018),Pages.1-4.

[13] Sanjeev Dewra, Rajinder Singh Kaler, "Placement of dynamic reconfigurable optical add/drop multiplexer using dynamic channel equalizer in an optical communication system," Optoelectronics and advanced materials-Rapid communications, (2015), Volume 9 no. 1-2, Pages 53-56. 